Claremont Colleges

Scholarship@ Claremont

All HMC Faculty Publications and Research

HMC Faculty Scholarship

$11-1-2006$

\title{
Summing Cubes by Counting Rectangles
}

Arthur T. Benjamin

Harvey Mudd College

Jennifer J. Quinn

University of Puget Sound

Calyssa Wurtz

Harvey Mudd College

\section{Recommended Citation}

Benjamin, Arthur T., Jennifer J. Quinn and Calyssa Wurtz. "Summing Cubes by Counting Rectangles." The College Mathematics Journal, November 2006.

This Article is brought to you for free and open access by the HMC Faculty Scholarship at Scholarship @ Claremont. It has been accepted for inclusion in All HMC Faculty Publications and Research by an authorized administrator of Scholarship @ Claremont. For more information, please contact scholarship@cuc.claremont.edu. 
Summing Cubes by Counting Rectangles

Author(s): Arthur T. Benjamin, Jennifer J. Quinn and Calyssa Wurtz

Source: The College Mathematics Journal, Vol. 37, No. 5 (Nov., 2006), pp. 387-389

Published by: Mathematical Association of America

Stable URL: http://www.jstor.org/stable/27646391

Accessed: $14 / 06 / 2013$ 16:37

Your use of the JSTOR archive indicates your acceptance of the Terms \& Conditions of Use, available at http://www.jstor.org/page/info/about/policies/terms.jsp

JSTOR is a not-for-profit service that helps scholars, researchers, and students discover, use, and build upon a wide range of content in a trusted digital archive. We use information technology and tools to increase productivity and facilitate new forms of scholarship. For more information about JSTOR, please contact support@ jstor.org. 


\section{Summing Cubes by Counting Rectangles}

Arthur T. Benjamin (benjamin@hmc.edu), Harvey Mudd College, Claremont, CA 91711, Jennifer J. Quinn (jquinn@awm-math.org), Association for Women in Mathematics, University of Puget Sound, Tacoma, WA 98416, and Calyssa Wurtz (wurcal@bethel.edu), Bethel University, St. Paul, MN 55112

Here is a beautiful combinatorial problem for your next homework assignment:

How many rectangles are contained in an $m \times n$ checkerboard?

(Hint: There is an elegant four-line proof.)

Many students will solve this problem by noticing that the number of $i \times j$ rectangles is $(m+1-i) \times(n+1-j)$ and hence the number of rectangles is

$$
\sum_{i=1}^{m} \sum_{j=1}^{n}(m+1-i)(n+1-j)=\left(\sum_{i=1}^{m} i\right)\left(\sum_{j=1}^{n} j\right)=\frac{m(m+1)}{2} \cdot \frac{n(n+1)}{2} .
$$

When your students ask about the four-line proof, explain that a rectangle is uniquely determined by choosing four lines, namely two horizontal lines and two vertical lines, which can be done in $\left(\begin{array}{c}m+1 \\ 2\end{array}\right)\left(\begin{array}{c}n+1 \\ 2\end{array}\right)$ ways. See Figure 1.

Figure 1. Every rectangle is determined by the choice of four lines.

But that's not the end of the story. As we'll see, we can apply this "checkerboard logic" to derive the famous formulas for summing squares and cubes. Recall that the sum of the first $n$ cubes is $\sum_{k=1}^{n} k^{3}=\left(\begin{array}{c}n+1 \\ 2\end{array}\right)^{2}$. This formula can be proved by induction, telescoping sums, combinatorially [1], or geometrically [2]. Our four-line proof suggests that we should be able to directly count rectangles on an $n \times n$ checkerboard and arrive at the same conclusion. But which rectangles are being counted by the $k^{3}$ term? We claim that these rectangles have upper right corner $(x, y)$ with largest coordinate $k$ (i.e., $\max \{x, y\}=k$ ). Such a rectangle would lie inside a $k \times k$ rectangle but not inside a $(k-1) \times(k-1)$ rectangle. See Figure 2.

From our four-line proof, there are

$$
\left(\begin{array}{c}
k+1 \\
2
\end{array}\right)^{2}-\left(\begin{array}{l}
k \\
2
\end{array}\right)^{2}=\frac{(k+1)^{2} k^{2}}{4}-\frac{k^{2}(k-1)^{2}}{4}=\frac{k^{2}}{4}\left((k+1)^{2}-(k-1)^{2}\right)=k^{3}
$$

such rectangles. But as combinatorial purists, we prefer to derive the $k^{3}$ term directlyavoiding algebra altogether. After all, the algebra presented is enough to directly prove the identity by telescoping sums. 
Figure 2. There are $k^{3}$ rectangles with maximum coordinate $k$.

Notice that there are $k^{3}$ ordered triples $(a, b, c)$ satisfying $0 \leq a, b, c<k$. Each of these triples can be paired to a rectangle with upper right corner $(x, y)$ having $\max \{x, y\}=k$ as illustrated in Figure 3 and described below:

- If $b \leq c,(a, b, c)$ is paired to the rectangle created from vertical lines $x=a$ and $x=k$ and horizontal line $y=b$ and $y=c+1$. The maximum coordinate of this rectangle equals $k$ and it occurs in the $x$-component.

- If $b>c,(a, b, c)$ is paired to the rectangle created from vertical lines $x=c$ and $x=b$, and horizontal lines $y=a$ and $y=k$. Here the maximum coordinate equals $k$ and it does not occur in the $x$-component since $c<b<k$.

Figure 3. The bijection between triple $(a, b, c)$ having $0 \leq a, b, c<k$ and rectangle having maximum coordinate equal $k$.

This process is easily reversed, since every rectangle with maximum coordinate equal to $k$ can be uniquely identified with a triple $(a, b, c)$ depending on whether the maximum occurs in the $x$-coordinate or not. A similar approach to this identity was given by Stein [3] who algebraically showed that there are $k^{3}$ rectangles with shorter side length equal to $n+1-k$ (for $1 \leq k \leq n$ ). We leave the reader with this challenge: 
For $1 \leq k \leq n$, find a four-line proof to show that there are $k^{3}$ rectangles on an $n \times n$ chessboard whose shorter side length is $n+1-k$. (Hint: How can you transform a rectangle with upper right corner $(x, y)$ having $\max \{x, y\}=k$ into a rectangle whose shorter side length equals $n+1-k$ ?)

Next we obtain a three-line proof for the sum of the squares, $\sum_{k=1}^{n} k^{2}$. Notice that $2\left(\begin{array}{c}n+1 \\ 3\end{array}\right)+\left(\begin{array}{c}n+1 \\ 2\end{array}\right)=n(n+1)(2 n+1) / 6$ is the familiar closed form for this sum. We now ask this question:

\section{How many squares exist in an $n \times n$ checkerboard?}

On the one hand, for $1 \leq k \leq n$, by considering the coordinates of the upper right corner, the number of $k \times k$ squares is $(n+1-k)(n+1-k)$. Hence the total number of squares is $\sum_{k=1}^{n}(n+1-k)^{2}=\sum_{k=1}^{n} k^{2}$.

On the other hand, for every ordered triple $(a, b, c)$ with $0 \leq a<b<c \leq n$ (there are $\left(\begin{array}{c}n+1 \\ 3\end{array}\right)$ of these), we create the square defined by the three lines $x=a, y=b$, and $y=c$. This generates all squares with lower left corner above the line $y=x$. Similarly, for the $\left(\begin{array}{c}n+1 \\ 3\end{array}\right)+\left(\begin{array}{c}n+1 \\ 2\end{array}\right)$ triples $(a, b, c)$ where $0 \leq b<a<c \leq n$ or $0 \leq b=a<c \leq n$, we use the lines $x=a, x=c$, and $y=b$ to generate the remaining squares with lower left corner on or below the line $y=x$. (See Figure 4.) Hence the number of squares is $2\left(\begin{array}{c}n+1 \\ 3\end{array}\right)+\left(\begin{array}{c}n+1 \\ 2\end{array}\right)$, as desired.

Figure 4. Counting squares yields $\sum_{k=1}^{n} k^{2}=2\left(\begin{array}{c}n+1 \\ 3\end{array}\right)+\left(\begin{array}{c}n+1 \\ 2\end{array}\right)$.

We conclude with the question of whether similar methods can be applied to sums of higher degree terms? We don't know-but we are counting on some reader to explore this question.

Acknowledgment. Thanks to Shai Simonson and Tim Woodcock for ideas that led to the three-line proof presented here. Thanks to Eric Gossett for directing the Bethel University summer research project "Visualizing Combinatorial Proofs" and Phil Kaasa for his skill coding java applets. The results of this project can be viewed at (www .mathcs . bethel .edu/ ${ }^{\sim}$ gossett/vcp/).

\section{References}

1. A. T. Benjamin and M. E. Orrison, Two Quick Combinatorial Proofs of $\sum_{k=1}^{n} k^{3}=\left(\begin{array}{c}n+1 \\ 2\end{array}\right)^{2}$, College Math. J., 33 (2002), 406-408.

2. R. B. Nelson, Proofs Without Words, Mathematical Association of America (1993), 84-90.

3. R. G. Stein, A Combinatorial Proof that $\sum k^{3}=\left(\sum k\right)^{2}$, Math. Mag., 44 (1971), 161-162. 